\title{
Fixed Point Results for Dualistic Contractions with an Application
}

\author{
Muhammad Nazam (D), Aiman Mukheimer $\mathbb{D}^{1},{ }^{2}$ Hassen Aydi $\mathbb{D}^{3},{ }^{3,4}$ Muhammad Arshad $\mathbb{D}^{5},{ }^{5}$ \\ and Raheel Riaz ${ }^{5}$
}

${ }^{1}$ Department of Mathematics, Allama Iqbal Open University, H-8, Islamabad 44000, Pakistan

${ }^{2}$ Université de Sousse, Institut Supérieur d'Informatique et des Techniques de Communication, H. Sousse 4000, Tunisia

${ }^{3}$ China Medical University Hospital, China Medical University, Taichung 40402, Taiwan

${ }^{4}$ Department of Mathematics and General Sciences, Prince Sultan University, Riyadh 11586, Saudi Arabia

${ }^{5}$ Department of Mathematics and Statistics, International Islamic University, H-10, Islamabad 44000, Pakistan

Correspondence should be addressed to Hassen Aydi; hassen.aydi@isima.rnu.tn

Received 10 June 2019; Revised 8 August 2019; Accepted 24 August 2019; Published 22 January 2020

Academic Editor: Yong Zhou

Copyright (c) 2020 Muhammad Nazam et al. This is an open access article distributed under the Creative Commons Attribution License, which permits unrestricted use, distribution, and reproduction in any medium, provided the original work is properly cited.

In this paper, by introducing a convergence comparison property of a self-mapping, we establish some new fixed point theorems for Bianchini type, Reich type, and Dass-Gupta type dualistic contractions defined on a dualistic partial metric space. Our work generalizes and extends some well known fixed point results in the literature. We also provide examples which show the usefulness of these dualistic contractions. As an application of our findings, we demonstrate the existence of the solution of an elliptic boundary value problem.

\section{Introduction}

The recent study in the metric fixed point theory is due to the Banach Contraction Principle, which has been modified, improved, and generalized in many ways in metric spaces (see for example, [1-17]).

This Contraction principle has also been studied in partial metric spaces (PMS) introduced by Matthews [18]. The PMS generalizes the metric space where the self-distance may be not equal to zero. The topological concepts, like convergence, Cauchy sequence, continuity, and completeness in this class can be found in [18-21] and references there in.

O'Neill [22] initiated the notion of a dualistic partial metric space. This class generalizes the notion of partial metric spaces. In [22], a relationship between a dualistic partial metric and a quasi metric has been obtained. O'Neill [22] also studied various topological properties of a dualistic partial metric space, while fixed point theory on dualistic partial metric spaces was presented by Oltra and Valero [23], who proved a Banach fixed point theorem and gave convergence properties of sequences on complete dualistic partial metric spaces. Later,
Nazam et al. [24] ensured fixed point results for rational type contraction mappings in this setting (see also the related paper [25]).

In this paper, motivated by $[26,27,12]$, we establish some new fixed point theorems in dualistic partial metric spaces, generalizing fixed point results of Bianchini [26], Reich [12], and Dass and Gupta [27]. We also provide examples and an application to show significance of the obtained results involving dualistic contractive conditions.

\section{Preliminaries}

We recall some mathematical basics and definitions.

Definition 1 ([18]). A partial metric on a non empty set $\mathscr{A}$ is a function $\eta: \mathscr{A} \times \mathscr{A} \rightarrow[0, \infty)$ so that

$$
\begin{aligned}
& \left(\eta_{1}\right) \quad \sigma=\varsigma \Longleftrightarrow \eta(\sigma, \sigma)=\eta(\sigma, \varsigma)=\eta(\varsigma, \varsigma) ; \\
& \left(\eta_{2}\right) \eta(\sigma, \sigma) \leq \eta(\sigma, \varsigma) ; \\
& \left(\eta_{3}\right) \eta(\sigma, \varsigma)=\eta(\varsigma, \sigma) ;
\end{aligned}
$$




$$
\left(\eta_{4}\right) \eta(\sigma, \varsigma) \leq \eta(\sigma, \xi)+\eta(\xi, \varsigma)-\eta(\xi, \xi)
$$

for all $\sigma, \varsigma, \xi \in \mathscr{A}$.

The pair $(\mathscr{A}, \eta)$ is called a partial metric space. O'Neill [22] did one significant change to the definition of the partial metric $\eta$ by extending its range from $[0, \infty)$ to $(-\infty, \infty)$. The partial metric $\eta$ with extended range is called a dualistic partial metric (DPM), denoted by $\eta^{*}$.

Definition 2 ([22]). Let $\mathscr{A}$ be a non empty set. If a function $\eta^{*}: \mathscr{A} \times \mathscr{A} \rightarrow \mathbb{R}$ is such that

$$
\begin{array}{ll}
\left(\eta_{1}^{*}\right) & \sigma=\varsigma \Longleftrightarrow \eta^{*}(\sigma, \sigma)=\eta^{*}(\varsigma, \varsigma)=\eta^{*}(\sigma, \varsigma) ; \\
\left(\eta_{2}^{*}\right) & \eta^{*}(\sigma, \sigma) \leq \eta^{*}(\sigma, \varsigma) ; \\
\left(\eta_{3}^{*}\right) & \eta^{*}(\sigma, \varsigma)=\eta^{*}(\varsigma, \sigma) ; \\
\left(\eta_{4}^{*}\right) \quad \eta^{*}(\sigma, \xi)+\eta^{*}(\varsigma, \varsigma) \leq \eta^{*}(\sigma, \varsigma)+\eta^{*}(\varsigma, \xi),
\end{array}
$$

for all $\sigma, \varsigma, \xi \in \mathscr{A}$, then $\eta^{*}$ is called a dualistic partial metric and the pair $\left(\mathscr{A}, \eta^{*}\right)$ is known as a dualistic partial metric space.

If $\left(\mathscr{A}, \eta^{*}\right)$ is a dualistic partial metric space, then $d_{\eta^{*}}: \mathcal{A} \times \mathcal{A} \rightarrow[0, \infty)$ defined by

$$
d_{\eta^{*}}(\sigma, \varsigma)=\eta^{*}(\sigma, \varsigma)-\eta^{*}(\sigma, \sigma), \quad \text { for all } \sigma, \varsigma \in \mathscr{A}
$$

is called a quasi metric on $\mathscr{A}$ such that $\tau\left(\eta^{*}\right)=\tau\left(d_{\eta^{*}}\right)$. Moreover, if $d_{\eta^{*}}$ is a dualistic quasi metric on $X$, then $d_{\eta^{*}}^{s}(\sigma, \varsigma)=\max \left\{d_{\eta^{*}}(\sigma, \varsigma), d_{\eta^{*}}(\varsigma, \sigma)\right\}$ is a metric on $\mathscr{A}$ (an induced metric).

Remark 1. Unlike partial metric case, note that if $\eta^{*}$ is a dualistic partial metric, then $\eta^{*}(\sigma, \varsigma)=0$ may not imply $\sigma=\varsigma$. The self-distance $\eta^{*}(\sigma, \sigma)$ is a feature utilized to describe the amount of information contained in $\sigma$. The restriction of $\eta^{*}$ to $[0, \infty)$ is a partial metric.

Example 1. We define $\eta^{*}: \mathbb{R} \times \mathbb{R} \rightarrow \mathbb{R}$ as $\eta^{*}(\sigma, \varsigma)=\max \{\sigma, \varsigma\}$. It is easy to check that $\eta^{*}$ satisfies $\left(\eta_{1}^{*}\right)-\left(\eta_{4}^{*}\right)$ and hence $\eta^{*}$ is a dualistic partial metric on $\mathbb{R}$. Mention that $\eta^{*}$ is not a partial metric on $\mathbb{R}$ because that $\eta^{*}(-m,-m)=-m$ for each $m>0$.

Example 2. Let $\eta$ be a partial metric defined on a non empty set $\mathscr{A}$. The function $\eta^{*}: \mathscr{A} \times \mathscr{A} \rightarrow \mathbb{R}$ defined by

$\eta^{*}(\sigma, \varsigma)=\eta(\sigma, \varsigma)-\eta(\sigma, \sigma)-\eta(\varsigma, \varsigma)$ for all $\sigma, \varsigma \in \mathscr{A}$

verifies $\left(\eta_{1}^{*}\right)-\left(\eta_{4}^{*}\right)$ and so it defines a dualistic partial metric on $\mathscr{A}$. Note that $\eta^{*}(\sigma, \varsigma)$ may have negative values.

In the following, a new example of a dualistic partial metric is stated.

Example 3. Take $\mathscr{A}=\mathbb{R}$ and $b>0$. We define $\eta^{*}: \mathscr{A} \times \mathscr{A} \rightarrow \mathbb{R}$ as

$$
\eta^{*}(\sigma, \varsigma)=\left\{\begin{array}{lll}
|\sigma-\varsigma| & \text { if } & \sigma \neq \varsigma \\
-b & \text { if } & \sigma=\varsigma .
\end{array}\right.
$$

The axioms $\left(\eta_{1}^{*}\right),\left(\eta_{2}^{*}\right)$, and $\left(\eta_{3}^{*}\right)$ can be proved immediately. We just prove axiom $\left(\eta_{4}^{*}\right)$ in details, for all $\sigma, \varsigma, v \in \mathscr{A}$.

Case 1. If $\sigma \neq \varsigma=v$, then $\eta(\sigma, v) \leq \eta(\sigma, \varsigma)+\eta(\varsigma, v)-\eta(\varsigma, \varsigma)$ implies $|\sigma-\nu|=|\sigma-\varsigma|$.

Case 2. If $\sigma=\varsigma \neq \nu$, then $\eta(\sigma, v) \leq \eta(\sigma, \varsigma)+\eta(\varsigma, v)-\eta(\varsigma, \varsigma)$ implies $|\sigma-v|=|\varsigma-v|$.

Case 3. If $\sigma=\varsigma=v$, then $\eta(\sigma, v) \leq \eta(\sigma, \varsigma)+\eta(\varsigma, v)-\eta(\varsigma, \varsigma)$ implies $-b=-b$.

Case 4. If $\sigma \neq \varsigma \neq v$, then $\eta(\sigma, v) \leq \eta(\sigma, \varsigma)+\eta(\varsigma, v)-\eta(\varsigma, \varsigma)$ implies $|\sigma-\nu| \leq|\sigma-\varsigma|+|\varsigma-\nu|+b$.

Thus, the axiom $\left(\eta_{4}^{*}\right)$ holds in all cases. Hence $\left(\mathscr{A}, \eta^{*}\right)$ is a dualistic partial metric space.

O'Neill [22] established that each dualistic partial metric $\eta^{*}$ on $\mathscr{A}$ generates a $T_{0}$ topology $\tau\left(\eta^{*}\right)$ on $\mathscr{A}$ having a base, the family of $\eta^{*}$-balls $\left\{B_{\eta^{*}}(\sigma, \epsilon): \sigma \in \mathscr{A}, \epsilon>0\right\}$ where

$$
B_{\eta^{*}}(\sigma, \epsilon)=\left\{\varsigma \in \mathscr{A}: \eta^{*}(\sigma, \varsigma)<\epsilon+\eta^{*}(\sigma, \sigma)\right\} .
$$

Definition 3 ([22] ). Let $\left(\mathscr{A}, \eta^{*}\right)$ be a dualistic partial metric space, then

(1) A sequence $\left\{\sigma_{n}\right\}_{n \in \mathbb{N}}$ in $\left(\mathscr{A}, \eta^{*}\right)$ converges to a point $\sigma \in \mathscr{A}$ if and only if $\eta^{*}(\sigma, \sigma)=\lim _{n \rightarrow \infty} \eta^{*}\left(\sigma, \sigma_{n}\right)$.

(2) A sequence $\left\{\sigma_{n}\right\}_{n \in \mathbb{N}}$ in $\left(\mathscr{A}, \eta^{*}\right)$ is called a Cauchy sequence if $\lim _{n, m \rightarrow \infty} \eta^{*}\left(\sigma_{n}, \sigma_{m}\right)$ exists and is finite.

(3) $\left(\mathscr{A}, \eta^{*}\right)$ is said to be complete if every Cauchy sequence $\left\{\sigma_{n}\right\}_{n \in \mathbb{N}}$ in $\mathscr{A}$ converges, with respect to $\tau\left(\eta^{*}\right)$, to a point $\sigma \in \mathscr{A}$ such that $\eta^{*}(\sigma, \sigma)=\lim _{n, m \rightarrow \infty} \eta^{*}\left(\sigma_{n}, \sigma_{m}\right)$.

The following lemma will be helpful in the sequel.

Lemma 1 ([22, 22]). (1) A dualistic partial metric space $\left(\mathscr{A}, \eta^{*}\right)$ is complete iff the metric space $\left(\mathscr{A}, d_{\eta^{*}}^{s}\right)$ is complete.

(2) A sequence $\left\{\sigma_{n}\right\}_{n \in \mathbb{N}}$ in $\mathscr{A}$ converges to a point $\sigma \in \mathscr{A}$, with respect to $\tau\left(d_{\eta^{*}}^{s}\right)$ iff

$$
\lim _{n \rightarrow \infty} \eta^{*}\left(\sigma, \sigma_{n}\right)=\eta^{*}(\sigma, \sigma)=\lim _{n, m \rightarrow \infty} \eta^{*}\left(\sigma_{n}, \sigma_{m}\right) .
$$

\section{Main Results}

We begin with the following useful property.

Definition 4. Let $T$ be a self-mapping on a dualistic partial metric space $\left(\mathscr{A}, \eta^{*}\right)$. If there is a convergent sequence $\left\{\sigma_{n}\right\}$ in $\mathscr{A}$ with $\sigma_{n} \rightarrow \sigma$, such that

$$
\eta^{*}(\sigma, \sigma) \leq \eta^{*}(T(\sigma), T(\sigma)),
$$

then $T$ is said to have the convergence comparison property [in short, (CCP)]. 
Example 4. Let $\mathscr{A}=\mathbb{Q}$. Define $\eta_{\vee}^{*}: \mathscr{A} \times \mathscr{A} \rightarrow \mathbb{R}$ by

$$
\eta_{\vee}^{*}(\sigma, \varsigma)=\max \{\sigma, \varsigma\} \quad \text { for all } \sigma, \varsigma \in \mathscr{A} .
$$

Clearly, $\left(\mathscr{A}, \eta_{\vee}^{*}\right)$ is a dualistic partial metric space. Consider $\left\{\sigma_{n}=1 / n-1, n \geq 1\right\}_{n \in \mathbb{N}} \subset \mathscr{A}$. We have $\lim _{n \rightarrow \infty} \eta_{\vee}^{*}\left(\sigma_{n},-1\right)=$ $\eta_{\vee}^{*}(-1,-1)$. That is, $\sigma_{n} \rightarrow \sigma=-1$ in $\left(\mathscr{A}, \eta_{\vee}^{*}\right)$. Define $T(u)=e^{u}$ for all $u \in \mathscr{A}$. For such $\sigma=-1$, observe that $\eta_{\vee}^{*}(\sigma, \sigma)=$ $-1 \leq e^{-1}=\eta_{\mathrm{V}}^{*}(T(\sigma), T(\sigma))$, so $T$ has the convergence comparison property.

The following theorem corresponds to the unique fixed point result of Bianchini type dualistic contraction.

Theorem 1. Let $T$ be a self-mapping on a complete dualistic partial metric space $\left(\mathscr{A}, \eta^{*}\right)$ satisfying $(C C P)$. If there is $k \in[0,1)$ so that

$$
\left|\eta^{*}(T(\sigma), T(\varsigma))\right| \leq k \max \left\{\left|\eta^{*}(\sigma, T(\sigma))\right|,\left|\eta^{*}(\varsigma, T(\varsigma))\right|\right\}
$$

for all $\sigma, \varsigma \in \mathscr{A}$, then $T$ possesses a unique fixed point.

Proof. We generate a Picard iterative sequence $\left\{\sigma_{n}\right\}_{n \in \mathbb{N}}$ with an initial point $\sigma_{0} \in \mathscr{A}$ such that $\sigma_{n}=T\left(\sigma_{n-1}\right)$ for all $n \in \mathbb{N}$. If there exists $n_{0} \in \mathbb{N}$ such that $\sigma_{n_{0}}=\sigma_{n_{0}+1}=T\left(\sigma_{n_{0}}\right)$, then $\sigma_{n_{0}}$ is a fixed point of $T$, so the proof is completed. From now on, assume that $\sigma_{n} \neq \sigma_{n+1}$ for all $n \in \mathbb{N}$, then by the contractive condition (8), we have

$$
\begin{aligned}
\left|\eta^{*}\left(\sigma_{n}, \sigma_{n+1}\right)\right| & =\left|\eta^{*}\left(T\left(\sigma_{n-1}\right), T\left(\sigma_{n}\right)\right)\right| \\
& \leq k \max \left\{\left|\eta^{*}\left(\sigma_{n-1}, T\left(\sigma_{n-1}\right)\right)\right|,\left|\eta^{*}\left(\sigma_{n}, T\left(\sigma_{n}\right)\right)\right|\right\} \\
& =k \max \left\{\left|\eta^{*}\left(\sigma_{n-1}, \sigma_{n}\right)\right|,\left|\eta^{*}\left(\sigma_{n}, \sigma_{n+1}\right)\right|\right\} .
\end{aligned}
$$

Thus,

$$
\left|\eta^{*}\left(\sigma_{n}, \sigma_{n+1}\right)\right| \leq k \max \left\{\left|\eta^{*}\left(\sigma_{n-1}, \sigma_{n}\right)\right|,\left|\eta^{*}\left(\sigma_{n}, \sigma_{n+1}\right)\right|\right\} .
$$

If

$$
\max \left\{\left|\eta^{*}\left(\sigma_{n-1}, \sigma_{n}\right)\right|,\left|\eta^{*}\left(\sigma_{n}, \sigma_{n+1}\right)\right|\right\}=\left|\eta^{*}\left(\sigma_{n}, \sigma_{n+1}\right)\right|,
$$

then (10) leads to a contradiction. As a result, we have

$$
\begin{aligned}
& \left|\eta^{*}\left(\sigma_{n}, \sigma_{n+1}\right)\right| \leq k\left|\eta^{*}\left(\sigma_{n-1}, \sigma_{n}\right)\right| \\
\left|\eta^{*}\left(\sigma_{n-1}, \sigma_{n}\right)\right| & =\left|\eta^{*}\left(T\left(\sigma_{n-2}\right), T\left(\sigma_{n-1}\right)\right)\right| \\
\leq & k \max \left\{\left|\eta^{*}\left(\sigma_{n-2}, T\left(\sigma_{n-2}\right)\right)\right|,\left|\eta^{*}\left(\sigma_{n-1}, T\left(\sigma_{n-1}\right)\right)\right|\right\} \\
& k \max \left\{\left|\eta^{*}\left(\sigma_{n-2}, \sigma_{n-1}\right)\right|,\left|\eta^{*}\left(\sigma_{n-1}, \sigma_{n}\right)\right|\right\}
\end{aligned}
$$

Arguing like above, we have

$\max \left\{\left|\eta^{*}\left(\sigma_{n-2}, \sigma_{n-1}\right)\right|,\left|\eta^{*}\left(\sigma_{n-1}, \sigma_{n}\right)\right|\right\}=\left|\eta^{*}\left(\sigma_{n-2}, \sigma_{n-1}\right)\right|$.

Thus, inequality (12) entails

$$
\left|\eta^{*}\left(\sigma_{n}, \sigma_{n+1}\right)\right| \leq k^{2}\left|\eta^{*}\left(\sigma_{n-2}, \sigma_{n-1}\right)\right| .
$$

Proceeding further in a similar way, we get

$$
\left|\eta^{*}\left(\sigma_{n}, \sigma_{n+1}\right)\right| \leq k^{n}\left|\eta^{*}\left(\sigma_{0}, \sigma_{1}\right)\right| \text {. }
$$

Now consider

$$
\begin{aligned}
\left|\eta^{*}\left(\sigma_{n}, \sigma_{n}\right)\right| & =\left|\eta^{*}\left(T\left(\sigma_{n-1}\right), T\left(\sigma_{n-1}\right)\right)\right| \\
& \leq k \max \left\{\left|\eta^{*}\left(\sigma_{n-1}, T\left(\sigma_{n-1}\right)\right)\right|,\left|\eta^{*}\left(\sigma_{n-1}, T\left(\sigma_{n-1}\right)\right)\right|\right\} \\
& =k \max \left\{\left|\eta^{*}\left(\sigma_{n-1}, \sigma_{n}\right)\right|,\left|\eta^{*}\left(\sigma_{n-1}, \sigma_{n}\right)\right|\right\}=k\left|\eta^{*}\left(\sigma_{n-1}, \sigma_{n}\right)\right|,
\end{aligned}
$$

the inequality (16) implies

$$
\left|\eta^{*}\left(\sigma_{n}, \sigma_{n}\right)\right| \leq k^{n}\left|\eta^{*}\left(\sigma_{0}, \sigma_{1}\right)\right| .
$$

We deduce from (1) that

$$
d_{\eta^{*}}\left(\sigma_{n}, \sigma_{n+1}\right)+\eta^{*}\left(\sigma_{n}, \sigma_{n}\right) \leq\left|\eta^{*}\left(\sigma_{n}, \sigma_{n+1}\right)\right|,
$$

and using inequalities (16) and (18), we obtain

$$
d_{\eta^{*}}\left(\sigma_{n}, \sigma_{n+1}\right) \leq 2 k^{n}\left|\eta^{*}\left(\sigma_{0}, \sigma_{1}\right)\right| .
$$

Now, for $m>n$, we have

$$
\begin{aligned}
d_{\eta^{*}}\left(\sigma_{n}, \sigma_{m}\right) \leq & d_{\eta^{*}}\left(\sigma_{n}, \sigma_{n+1}\right)+d_{\eta^{*}}\left(\sigma_{n+1}, \sigma_{n+2}\right) \\
& +\cdots+d_{\eta^{*}}\left(\sigma_{m-1}, \sigma_{m}\right) \\
\leq & 2 k^{n}\left|\eta^{*}\left(\sigma_{0}, \sigma_{1}\right)\right|+2 k^{n+1}\left|\eta^{*}\left(\sigma_{0}, \sigma_{1}\right)\right| \\
& +\cdots+2 k^{m-1}\left|\eta^{*}\left(\sigma_{0}, \sigma_{1}\right)\right| \\
\leq & 2\left(k^{n}+k^{n+1}+\cdots+k^{m-1}\right)\left|\eta^{*}\left(\sigma_{0}, \sigma_{1}\right)\right| \\
\leq & 2 \frac{k^{n}\left(1-k^{m-n}\right)}{1-k}\left|\eta^{*}\left(\sigma_{0}, \sigma_{1}\right)\right|
\end{aligned}
$$

$$
\begin{aligned}
d_{\eta^{*}}\left(\sigma_{m}, \sigma_{n}\right) \leq & d_{\eta^{*}}\left(\sigma_{m}, \sigma_{m-1}\right)+d_{\eta^{*}}\left(\sigma_{m-1}, \sigma_{m-2}\right) \\
& +\cdots+d_{\eta^{*}}\left(\sigma_{n+1}, \sigma_{n}\right) \\
\leq & 2 k^{m-1}\left|\eta^{*}\left(\sigma_{0}, \sigma_{1}\right)\right|+2 k^{m-2}\left|\eta^{*}\left(\sigma_{0}, \sigma_{1}\right)\right| \\
& +\cdots+2 k^{n}\left|\eta^{*}\left(\sigma_{0}, \sigma_{1}\right)\right| \\
\leq & 2\left(k^{n}+k^{n+1}+\cdots+k^{m-1}\right)\left|\eta^{*}\left(\sigma_{0}, \sigma_{1}\right)\right| \\
\leq & 2 \frac{k^{n}\left(1-k^{m-n}\right)}{1-k}\left|\eta^{*}\left(\sigma_{0}, \sigma_{1}\right)\right| .
\end{aligned}
$$

As $m, n \rightarrow \infty, d_{\eta^{*}}^{s}\left(\sigma_{n}, \sigma_{m}\right)=\max \left\{d_{\eta}^{*}\left(\sigma_{n}, \sigma_{m}\right), d_{\eta}^{*}\left(\sigma_{m}, \sigma_{n}\right)\right\} \rightarrow 0$, thus, $\left\{\sigma_{n}\right\}$ is a Cauchy sequence in $\left(\mathscr{A}, d_{\eta^{*}}^{s}\right)$. Since $\left(\mathscr{A}, \eta^{*}\right)$ is a complete dualistic partial metric space, by Lemma 1 (1), $\left(\mathscr{A}, d_{\eta^{*}}^{s}\right)$ is a complete metric space. Thus, there exists $\sigma^{*} \in\left(\mathscr{A}, d_{\eta^{*}}^{s}\right)$ such that $\sigma_{n} \rightarrow \sigma^{*}$ as $n \rightarrow \infty$, that is $\lim _{n \rightarrow \infty} d_{\eta^{*}}\left(\sigma_{n}, \sigma^{*}\right)=0$ and by Lemma 1 (2), we know that

$$
\eta^{*}\left(\sigma^{*}, \sigma^{*}\right)=\lim _{n \rightarrow \infty} \eta^{*}\left(\sigma_{n}, \sigma^{*}\right)=\lim _{n, m \rightarrow \infty} \eta^{*}\left(\sigma_{n}, \sigma_{m}\right) .
$$

Since, $\lim _{m, n \rightarrow \infty} d_{\eta^{*}}\left(\sigma_{m}, \sigma_{n}\right)=0$, by (1) and (18), we have

$$
\eta^{*}\left(\sigma^{*}, \sigma^{*}\right)=\lim _{n \rightarrow \infty} \eta^{*}\left(\sigma_{n}, \sigma^{*}\right)=\lim _{n, m \rightarrow \infty} \eta^{*}\left(\sigma_{n}, \sigma_{m}\right)=0 .
$$


This shows that $\left\{\sigma_{n}\right\}$ is a Cauchy sequence converging to $\sigma^{*} \in\left(\mathscr{A}, \eta^{*}\right)$. We shall show that $\sigma^{*}$ is a fixed point of $T$. By (8), we have

$$
\begin{aligned}
\left|\eta^{*}\left(\sigma_{n}, T\left(\sigma^{*}\right)\right)\right| & =\left|\eta^{*}\left(T\left(\sigma_{n-1}\right), T\left(\sigma^{*}\right)\right)\right| \\
& \leq k \max \left\{\left|\eta^{*}\left(\sigma_{n-1}, T\left(\sigma_{n-1}\right)\right)\right|,\left|\eta^{*}\left(\sigma^{*}, T\left(\sigma^{*}\right)\right)\right|\right\},
\end{aligned}
$$

as $n \rightarrow \infty,\left|\eta^{*}\left(\sigma^{*}, T\left(\sigma^{*}\right)\right)\right| \leq k\left|\eta^{*}\left(\sigma^{*}, T\left(\sigma^{*}\right)\right)\right|$ which implies that $\eta^{*}\left(\sigma^{*}, T\left(\sigma^{*}\right)\right)=0$. Since $T$ has (CCP), we get

$$
0=\eta^{*}\left(\sigma^{*}, \sigma^{*}\right) \leq \eta^{*}\left(T\left(\sigma^{*}\right), T\left(\sigma^{*}\right)\right) .
$$

On the other hand, by axiom $\left(\eta_{4}^{*}\right)$, we have $\eta^{*}\left(\sigma^{*}, \sigma^{*}\right) \leq$ $\eta^{*}\left(\sigma^{*}, T\left(\sigma^{*}\right)\right)+\eta^{*}\left(T\left(\sigma^{*}\right), \sigma^{*}\right)-\eta^{*}\left(T\left(\sigma^{*}\right), T\left(\sigma^{*}\right)\right)$ which implies that

$$
\eta^{*}\left(T\left(\sigma^{*}\right), T\left(\sigma^{*}\right)\right) \leq 0 .
$$

The inequalities (26) and (27) imply that $\eta^{*}\left(T\left(\sigma^{*}\right)\right.$, $\left.T\left(\sigma^{*}\right)\right)=0$. Thus,

$$
\eta^{*}\left(\sigma^{*}, T\left(\sigma^{*}\right)\right)=\eta^{*}\left(T\left(\sigma^{*}\right), T\left(\sigma^{*}\right)\right)=\eta^{*}\left(\sigma^{*}, \sigma^{*}\right) .
$$

By using axiom $\left(\eta_{1}^{*}\right)$, we have $\sigma^{*}=T\left(\sigma^{*}\right)$. This shows that $\sigma^{*}$ is a fixed point of $T$. To prove the uniqueness, suppose that $y^{*}$ is another fixed point of $T$, then $T\left(y^{*}\right)=y^{*}$ and $\eta^{*}\left(y^{*}, y^{*}\right)=0$. By (8), we obtain

$$
\begin{aligned}
\left|\eta^{*}\left(\sigma^{*}, y^{*}\right)\right| & =\left|\eta^{*}\left(T\left(\sigma^{*}\right), T\left(y^{*}\right)\right)\right| \\
& \leq k \max \left\{\left|\eta^{*}\left(\sigma^{*}\right),\left(T\left(\sigma^{*}\right)\right)\right|,\left|\eta^{*}\left(y^{*}, T\left(y^{*}\right)\right)\right|\right\}=0 .
\end{aligned}
$$

This implies that $\eta^{*}\left(\sigma^{*}, y^{*}\right)=\eta^{*}\left(\sigma^{*}, \sigma^{*}\right)=\eta^{*}\left(y^{*}, y^{*}\right)=0$ and using axiom $\left(\eta_{1}^{*}\right)$, we have $\sigma^{*}=y^{*}$, which proves the uniqueness of $\sigma^{*}$.

Corollary 1. Let $(\mathscr{A}, \eta)$ be a complete partial metric space and let $T: \mathscr{A} \rightarrow \mathscr{A}$ be a mapping satisfying

$$
\eta(T(\sigma), T(\varsigma)) \leq k \max \{\eta(\sigma, T(\sigma)), \eta(\varsigma, T(\varsigma))\},
$$

for all $\sigma, \varsigma \in \mathscr{A}$ and $0 \leq k<1$. Then, Thas a unique fixed point.

Proof. Since the restriction of a dualistic partial metric $\eta^{*}$ to $[0, \infty)$, which is $\left.\eta^{*}\right|_{[0, \infty)}=\eta$, is a partial metric, so arguments follow the same lines as in the proof of Theorem 1 .

Corollary 2 ([26]). Let $(\mathscr{A}, d)$ be a complete metric space and let $T: \mathscr{A} \rightarrow \mathscr{A}$ be a mapping satisfying

$$
d(T(\sigma), T(\varsigma)) \leq k \max \{d(\sigma, T(\sigma)), d(\varsigma, T(\varsigma))\},
$$

for all $\sigma, \varsigma \in \mathscr{A}$ and $0 \leq k<1$. Then, T has a unique fixed point.

Proof. Set $\eta(\sigma, \sigma)=0$ for all $\sigma \in \mathscr{A}$, in Corollary 1 .

Example 5. Let $\mathscr{A}=(-\infty, 0]$. Define the mapping $\eta^{*}: \mathscr{A} \times \mathscr{A} \rightarrow \mathbb{R}$ by

$$
\eta^{*}(\sigma, \varsigma)=\left\{\begin{array}{lll}
|\sigma-\varsigma| & \text { if } & \sigma \neq \varsigma, \\
\sigma \vee \varsigma & \text { if } & \sigma=\varsigma,
\end{array}\right.
$$

then, $\left(\mathscr{A}, \eta^{*}\right)$ is a complete dualistic partial metric space. Define a mapping $T: \mathscr{A} \rightarrow \mathscr{A}$ by

$$
T(\sigma)=\left\{\begin{array}{lll}
-1 & \text { if } & \sigma \in(-\infty,-4] \\
0 & \text { if } & \sigma \in(-4,0] .
\end{array}\right.
$$

Note that the mapping $T$ has the convergence comparison property (CCP). Indeed, consider $\left\{\sigma_{n}=-1 / n-2 \mid n=1,2, \ldots\right\}$. Here $\left\{\sigma_{n}\right\}$ is a sequence in $\mathscr{A}$. Clearly, we have that $\sigma_{n} \rightarrow \sigma=-2$ as $n \rightarrow \infty$ in $\left(\mathscr{A}, \eta^{*}\right)$. We have $\eta^{*}(-2,-2)=-2 \leq=0$ $=\eta^{*}(T(-2), T(-2))$. Choose $k=3 / 4$. On the other hand, the contractive condition (8) is also satisfied. For this, let $\sigma, \varsigma \in \mathscr{A}$. Here, we need the following cases:

Case 1. (a) $\sigma \neq \varsigma$ and $\sigma, \varsigma \in(-4,0]$. We have

$$
\left|\eta^{*}(T(\sigma), T(\varsigma))\right|=0 \text {. }
$$

(b) $\sigma \neq \varsigma$ and $\sigma, \varsigma \in(-\infty,-4]$. We have

$\left|\eta^{*}(T(\sigma), T(\varsigma))\right|=1 \leq 3 k \leq k \max \left\{\left|\eta^{*}(\sigma, T(\sigma))\right|,\left|\eta^{*}(\varsigma, T(\varsigma))\right|\right\}$.

(c) $\sigma \neq \varsigma$ and $\sigma \in(-\infty,-4], \varsigma \in(-4,0]$ and vice versa. In this case,

$\left|\eta^{*}(T(\sigma), T(\varsigma))\right|=1 \leq 3 k \leq k \max \left\{\left|\eta^{*}(\sigma, T(\sigma))\right|,\left|\eta^{*}(\varsigma, T(\varsigma))\right|\right\}$.

Case 2. (a) $\sigma=\varsigma \in(-4,0]$. We have

$$
\left|\eta^{*}(T(\sigma), T(\varsigma))\right|=0 .
$$

(b) $\sigma=\varsigma \in(-\infty,-4]$. We have

$\left|\eta^{*}(T(\sigma), T(\varsigma))\right|=1 \leq 3 k \leq k \max \left\{\left|\eta^{*}(\sigma, T(\sigma))\right|,\left|\eta^{*}(\varsigma, T(\varsigma))\right|\right\}$.

Hence, $T$ verifies all conditions of Theorem 1 . Note that $\sigma=0$ is the unique fixed point of $T$.

Example 6. Let $\mathscr{A}=(-\infty, 0]$. Define the mapping $\eta^{*}: \mathscr{A} \times \mathscr{A} \rightarrow \mathbb{R}$ by

$$
\eta^{*}(\sigma, \varsigma)=\left\{\begin{array}{lll}
\sigma+\varsigma & \text { if } & \sigma \neq \varsigma \\
\sigma \vee \varsigma & \text { if } & \sigma=\varsigma
\end{array}\right.
$$

then $\left(\mathscr{A}, \eta^{*}\right)$ is a complete dualistic partial metric space. Define a mapping $T: \mathscr{A} \rightarrow \mathscr{A}$ as in Example 5. It is obvious that $T$ verifies all conditions of Theorem 1. But, classical Bianchini contraction (contraction without the absolute value function) is not applicable. Indeed, for $\sigma=-3$ and $\varsigma=-2$, we have

$\eta^{*}(T(\sigma), T(\varsigma))=\eta^{*}(0,0)=0>-2 k=k \max \left\{\eta^{*}(\sigma, T(\sigma)), \eta^{*}(\varsigma, T(\varsigma))\right\}$

for each $k \in(0,1)$.

In what follows, we investigate a unique fixed point of Reich type dualistic contraction mappings. 
Theorem 2. Let $\left(\mathscr{A}, \eta^{*}\right)$ be a complete dualistic partial metric space and let $T: \mathscr{A} \rightarrow \mathscr{A}$ be a mapping satisfying (CCP). If there exist $a, b, c \geq 0$ with $a+b+c<1$ such that

$\left|\eta^{*}(T(\sigma), T(\varsigma))\right| \leq a\left|\eta^{*}(\sigma, y)\right|+b\left|\eta^{*}(\sigma, T(\sigma))+c\right| \eta^{*}(\varsigma, T(\varsigma)) \mid$

for all $\sigma, \varsigma \in \mathscr{A}$, then, $T$ has a fixed point.

Proof. Let $\left\{\sigma_{n}\right\}_{n \in \mathbb{N}}$ be a sequence with an initial point $\sigma_{0} \in X$ such that $\sigma_{n}=T\left(\sigma_{n-1}\right)$ for all $n \in \mathbb{N}$. If there exists $n_{0} \in \mathbb{N}$ such that $\sigma_{n_{0}}=\sigma_{n_{0}+1}=T\left(\sigma_{n_{0}}\right)$, then $\sigma_{n_{0}}$ is a fixed point of $T$. On the other hand, if $\sigma_{n} \neq \sigma_{n+1}$ for all $n \in \mathbb{N}$, then by contractive condition (41), we have

$$
\begin{aligned}
\left|\eta^{*}\left(\sigma_{n}, \sigma_{n+1}\right)\right|= & \left|\eta^{*}\left(T\left(\sigma_{n-1}\right), T\left(\sigma_{n}\right)\right)\right| \\
\leq & a\left|\eta^{*}\left(\sigma_{n-1}, \sigma_{n}\right)\right|+b\left|\eta^{*}\left(\sigma_{n-1}, T\left(\sigma_{n-1}\right)\right)\right| \\
& +c\left|\eta^{*}\left(\sigma_{n}, T\left(\sigma_{n}\right)\right)\right| \\
= & a\left|\eta^{*}\left(\sigma_{n-1}, \sigma_{n}\right)\right|+b\left|\eta^{*}\left(\sigma_{n-1}, \sigma_{n}\right)\right|+c\left|\eta^{*}\left(\sigma_{n}, \sigma_{n+1}\right)\right|,
\end{aligned}
$$

$$
\left|\eta^{*}\left(\sigma_{n}, \sigma_{n+1}\right)\right| \leq \frac{a+b}{1-c}\left|\eta^{*}\left(\sigma_{n-1}, \sigma_{n}\right)\right| .
$$

Let $k=(a+b) /(1-c)$, then $0 \leq k<1$ and repeating arguments given above, we have

$$
\left|\eta^{*}\left(\sigma_{n}, \sigma_{n+1}\right)\right| \leq k^{n}\left|\eta^{*}\left(\sigma_{0}, \sigma_{1}\right)\right| .
$$

Now, consider the self-distance

$$
\begin{aligned}
\left|\eta^{*}\left(\sigma_{n}, \sigma_{n}\right)\right|= & \left|\eta^{*}\left(T\left(\sigma_{n-1}\right), T\left(\sigma_{n-1}\right)\right)\right| \leq a\left|\eta^{*}\left(\sigma_{n-1}, \sigma_{n-1}\right)\right| \\
& +b\left|\eta^{*}\left(\sigma_{n-1}, T\left(\sigma_{n-1}\right)\right)\right|+c\left|\eta^{*}\left(\sigma_{n-1}, T\left(\sigma_{n-1}\right)\right)\right| \\
= & a\left|\eta^{*}\left(\sigma_{n-1}, \sigma_{n-1}\right)\right|+b\left|\eta^{*}\left(\sigma_{n-1}, \sigma_{n}\right)\right|+c\left|\eta^{*}\left(\sigma_{n-1}, \sigma_{n}\right)\right| \\
= & a\left|\eta^{*}\left(\sigma_{n-1}, \sigma_{n-1}\right)\right|+(b+c)\left|\eta^{*}\left(\sigma_{n-1}, \sigma_{n}\right)\right| .
\end{aligned}
$$

Due to inequality (44), we have

$$
\left|\eta^{*}\left(\sigma_{n}, \sigma_{n}\right)\right| \leq a\left|\eta^{*}\left(\sigma_{n-1}, \sigma_{n-1}\right)\right|+(b+c) k^{n-1}\left|\eta^{*}\left(\sigma_{0}, \sigma_{1}\right)\right|,
$$

$$
\begin{aligned}
\left|\eta^{*}\left(\sigma_{n-1}, \sigma_{n-1}\right)\right|= & \left|\eta^{*}\left(T\left(\sigma_{n-2}\right), T\left(\sigma_{n-2}\right)\right)\right| \leq a\left|\eta^{*}\left(\sigma_{n-2}, \sigma_{n-2}\right)\right| \\
& +b\left|\eta^{*}\left(\sigma_{n-2}, T\left(\sigma_{n-2}\right)\right)\right|+c\left|\eta^{*}\left(\sigma_{n-2}, T\left(\sigma_{n-2}\right)\right)\right| \\
\leq & a\left|\eta^{*}\left(\sigma_{n-2}, \sigma_{n-2}\right)\right|+(b+c)\left|\eta^{*}\left(\sigma_{n-2}, \sigma_{n-1}\right)\right| \\
\leq & a\left|\eta^{*}\left(\sigma_{n-2}, \sigma_{n-2}\right)\right|+(b+c) k^{n-2}\left|\eta^{*}\left(\sigma_{0}, \sigma_{1}\right)\right| .
\end{aligned}
$$

The inequality (46) implies that

$$
\begin{aligned}
\left|\eta^{*}\left(\sigma_{n}, \sigma_{n}\right)\right| \leq & a^{2}\left|\eta^{*}\left(\sigma_{n-2}, \sigma_{n-2}\right)\right|+a(b+c) k^{n-2}\left|\eta^{*}\left(\sigma_{0}, \sigma_{1}\right)\right| \\
& +(b+c) k^{n-1}\left|\eta^{*}\left(\sigma_{0}, \sigma_{1}\right)\right| \leq a^{3}\left|\eta^{*}\left(\sigma_{n-3}, \sigma_{n-3}\right)\right| \\
& +\left(a^{2} k^{n-3}+a k^{n-2}+k^{n-1}\right)(b+c)\left|\eta^{*}\left(\sigma_{0}, \sigma_{1}\right)\right| .
\end{aligned}
$$

Continuing further, we get

$$
\begin{aligned}
\left|\eta^{*}\left(\sigma_{n}, \sigma_{n}\right)\right| \leq & a^{n}\left|\eta^{*}\left(\sigma_{0}, \sigma_{0}\right)\right|+\left(a^{n-1}+a^{n-2} k+a^{n-3} k^{2}\right. \\
& \left.+\cdots+a k^{n-2}+k^{n-1}\right)(b+c)\left|\eta^{*}\left(\sigma_{0}, \sigma_{1}\right)\right|
\end{aligned}
$$

$\left|\eta^{*}\left(\sigma_{n}, \sigma_{n}\right)\right| \leq a^{n}\left|\eta^{*}\left(\sigma_{0}, \sigma_{0}\right)\right|+\left(\frac{k^{n}-a^{n}}{k-a}\right)(b+c)\left|\eta^{*}\left(\sigma_{0}, \sigma_{1}\right)\right|$.

The equation (1) implies that

$$
\begin{aligned}
d_{\eta^{*}}\left(\sigma_{n}, \sigma_{n+1}\right) \leq & \left|\eta^{*}\left(\sigma_{n}, \sigma_{n+1}\right)\right|-\eta^{*}\left(\sigma_{n}, \sigma_{n}\right) \leq\left|\eta^{*}\left(\sigma_{n}, \sigma_{n+1}\right)\right| \\
& +\left|\eta^{*}\left(\sigma_{n}, \sigma_{n}\right)\right| \leq k^{n}\left|\eta^{*}\left(\sigma_{0}, \sigma_{1}\right)\right|+a^{n}\left|\eta^{*}\left(\sigma_{0}, \sigma_{0}\right)\right| \\
& +\frac{k^{n}-a^{n}}{k-a}(b+c)\left|\eta^{*}\left(\sigma_{0}, \sigma_{1}\right)\right| \\
\leq & \left(k^{n}+(b+c)\left(\frac{k^{n}-a^{n}}{k-a}\right)\right)\left|\eta^{*}\left(\sigma_{0}, \sigma_{1}\right)\right|+a^{n}\left|\eta^{*}\left(\sigma_{0}, \sigma_{0}\right)\right| \\
\leq & \left(k^{n}+\frac{k^{n}-a^{n}}{k-a}\right)\left|\eta^{*}\left(\sigma_{0}, \sigma_{1}\right)\right|+a^{n}\left|\eta^{*}\left(\sigma_{0}, \sigma_{0}\right)\right| \\
= & \left(\frac{k^{n}(k-a)+k^{n}-a^{n}}{k-a}\right)\left|\eta^{*}\left(\sigma_{0}, \sigma_{1}\right)\right|+a^{n}\left|\eta^{*}\left(\sigma_{0}, \sigma_{0}\right)\right| \\
= & \left(k^{n}+k^{n-1}+a k^{n-2}+\cdots+a^{n-1}\right)\left|\eta^{*}\left(\sigma_{0}, \sigma_{1}\right)\right|+a^{n}\left|\eta^{*}\left(\sigma_{0}, \sigma_{0}\right)\right| .
\end{aligned}
$$

Let $\rho^{n}=k^{n}+k^{n-1}+a k^{n-2}+\cdots+a^{n-1}$. Then,

$$
d_{\eta^{*}}\left(\sigma_{n}, \sigma_{n+1}\right) \leq \rho^{n}\left|\eta^{*}\left(\sigma_{0}, \sigma_{1}\right)\right|+a^{n}\left|\eta^{*}\left(\sigma_{0}, \sigma_{0}\right)\right| .
$$

Now, for $m>n$, we have

$$
\begin{aligned}
d_{\eta^{*}}\left(\sigma_{n}, \sigma_{m}\right) \leq & d_{\eta^{*}}\left(\sigma_{n}, \sigma_{n+1}\right)+d_{\eta^{*}}\left(\sigma_{n+1}, \sigma_{n+2}\right)+\cdots+d_{\eta^{*}}\left(\sigma_{m-1}, \sigma_{m}\right) \\
\leq & \rho^{n}\left|\eta^{*}\left(\sigma_{0}, \sigma_{1}\right)\right|+a^{n}\left|\eta^{*}\left(\sigma_{0}, \sigma_{0}\right)\right|+\rho^{n+1}\left|\eta^{*}\left(\sigma_{0}, \sigma_{1}\right)\right| \\
& +a^{n+1}\left|\eta^{*}\left(\sigma_{0}, \sigma_{0}\right)\right| \\
& +\cdots+\rho^{m-1}\left|\eta^{*}\left(\sigma_{0}, \sigma_{1}\right)\right|+a^{m-1}\left|\eta^{*}\left(\sigma_{0}, \sigma_{0}\right)\right| \\
= & \left(\rho^{n}+\rho^{n+1}+\cdots+\rho^{n+1}\right)\left|\eta^{*}\left(\sigma_{0}, \sigma_{1}\right)\right| \\
& +\left(a^{n}+a^{n+1}+\cdots+a^{m-1}\right)\left|\eta^{*}\left(\sigma_{0}, \sigma_{0}\right)\right| \\
\leq & \left(\rho^{n}+\rho^{n+1}+\cdots+\rho^{n+1}+\cdots\right)\left|\eta^{*}\left(\sigma_{0}, \sigma_{1}\right)\right| \\
& +\left(a^{n}+a^{n+1}+\cdots+a^{m-1}+\cdots\right)\left|\eta^{*}\left(\sigma_{0}, \sigma_{0}\right)\right| \\
= & \frac{\rho^{n}}{1-\rho}\left|\eta^{*}\left(\sigma_{0}, \sigma_{1}\right)\right|+\frac{a^{n}}{1-a}\left|\eta^{*}\left(\sigma_{0}, \sigma_{0}\right)\right| .
\end{aligned}
$$

We conclude that $\lim _{m, n \rightarrow \infty} d_{\eta^{*}}^{s}\left(\sigma_{n}, \sigma_{m}\right)=0$. This shows that $\left\{\sigma_{n}\right\}$ is a Cauchy sequence in $\left(\mathscr{A}, d_{\eta^{*}}^{s}\right)$. Since $\left(\mathscr{A}, \eta^{*}\right)$ is a complete dualistic partial metric space, by Lemma $1(1),\left(\mathscr{A}, d_{\eta^{*}}^{s}\right)$ is a complete metric space. Thus, there exists $j^{*} \in\left(\mathscr{A}, d_{\eta^{*}}^{s}\right)$ such that $\sigma_{n} \rightarrow j^{*}$ as $n \rightarrow \infty$, that is $\lim _{n \rightarrow \infty} d_{\eta^{*}}\left(\sigma_{n}, j^{*}\right)=0$ and by Lemma 1 (2), we know that

$$
\eta^{*}\left(j^{*}, j^{*}\right)=\lim _{n \rightarrow \infty} \eta^{*}\left(\sigma_{n}, j^{*}\right)=\lim _{n, m \rightarrow \infty} \eta^{*}\left(\sigma_{n}, \sigma_{m}\right) .
$$

Since $\lim _{m, n \rightarrow \infty} d_{\eta^{*}}\left(\sigma_{m}, \sigma_{n}\right)=0$, by (1) and (50), we have

$$
\eta^{*}\left(j^{*}, j^{*}\right)=\lim _{n \rightarrow \infty} \eta^{*}\left(\sigma_{n}, j^{*}\right)=\lim _{n, m \rightarrow \infty} \eta^{*}\left(\sigma_{n}, \sigma_{m}\right)=0 .
$$

This shows that $\left\{\sigma_{n}\right\}$ is a Cauchy sequence converging to $j^{*} \in\left(\mathscr{A}, \eta^{*}\right)$. We show that $j^{*}$ is a fixed point of $T$. By (41), we have

$$
\begin{aligned}
\left|\eta^{*}\left(\sigma_{n}, T\left(j^{*}\right)\right)\right|= & \left|\eta^{*}\left(T\left(\sigma_{n-1}\right), T\left(j^{*}\right)\right)\right| \leq a\left|\eta^{*}\left(\sigma_{n-1}, j^{*}\right)\right| \\
& +b\left|\eta^{*}\left(\sigma_{n-1}, T\left(\sigma_{n-1}\right)\right)\right|+c\left|\eta^{*}\left(j^{*}, T\left(j^{*}\right)\right)\right| .
\end{aligned}
$$

Applying limit as $n \rightarrow \infty$ and using equation (55), we have $\eta^{*}\left(j^{*}, T\left(j^{*}\right)\right)=0$. Since $T$ has (CCP), we get 
TABLE 1: Evaluation of contractive condition.

\begin{tabular}{|c|c|c|}
\hline$(\sigma, \varsigma)$ & $\left|\eta^{*}(T(\sigma), T(\varsigma))\right|$ & $a\left|\eta^{*}(\sigma, y)\right|+b\left|\eta^{*}(\sigma, T(\sigma))\right|+c\left|\eta^{*}(\varsigma, T(\varsigma))\right|$ \\
\hline$(0,0)$ & 0 & 0 \\
\hline$(0,-1)$ & 0 & $a+c$ \\
\hline$(0,-2)$ & 0.3 & $2 a+1.7 c$ \\
\hline$(0,-3)$ & 0.1 & $3 a+2.9 c$ \\
\hline$(0,-0.3)$ & 0 & $0.3(a+c)$ \\
\hline$(0,-0.1)$ & 0 & $0.1(a+c)$ \\
\hline$(-1,-2)$ & 0.3 & $a+b+2 c$ \\
\hline$(-1,-3)$ & 0.1 & $2 a+b+2.9 c$ \\
\hline$(-1,-0.1)$ & 0 & $0.9 a+b+0.1 c$ \\
\hline$(-1,-0.3)$ & 0 & $0.7 a+b+0.3 c$ \\
\hline$(-1,-1)$ & 0 & $a+b+c$ \\
\hline$(-2,-2)$ & 0.3 & $2 a+1.7(b+c)$ \\
\hline$(-2,-3)$ & 0.2 & $a+1.7 b+2.9 c$ \\
\hline$(-2,-0.3)$ & 0.3 & $1.7(a+b)+0.3 c$ \\
\hline$(-2,-0.1)$ & 0.3 & $1.9 a+1.7 b+0.1 c$ \\
\hline$(-3,-3)$ & 0.1 & $3 a+2.9(b+c)$ \\
\hline$(-3,-0.1)$ & 0.1 & $2.9(a+b)+0.1 c$ \\
\hline$(-3,-0.3)$ & 0.1 & $2.7 a+2.9 b+0.3 c$ \\
\hline$(-0.3,-0.3)$ & 0 & $0.3(a+b+c)$ \\
\hline$(-0.1,-0.1)$ & 0 & $0.1(a+b+c)$ \\
\hline
\end{tabular}

$$
0=\eta^{*}\left(j^{*}, j^{*}\right) \leq \eta^{*}\left(T\left(j^{*}\right), T\left(j^{*}\right)\right) .
$$

By axiom $\left(\eta_{2}^{*}\right)$, we have

$$
\eta^{*}\left(T\left(j^{*}\right), T\left(j^{*}\right)\right) \leq \eta^{*}\left(j^{*}, T\left(j^{*}\right)\right)=0 .
$$

The inequalities (57) and (58) imply that $\eta^{*}\left(T\left(j^{*}\right), T\left(j^{*}\right)\right)=0$. Thus,

$$
\eta^{*}\left(j^{*}, T\left(j^{*}\right)\right)=\eta^{*}\left(T\left(j^{*}\right), T\left(j^{*}\right)\right)=\eta^{*}\left(j^{*}, j^{*}\right) .
$$

By using axiom $\left(\eta_{1}^{*}\right)$, we have $j^{*}=T\left(j^{*}\right)$. This shows that $j^{*}$ is a fixed point of $T$.

Example 7. Let $\mathscr{A}=\{0,-1,-2,-3,-0.3,-0.1\} \quad$ and $\eta^{*}: \mathscr{A} \times \mathscr{A} \rightarrow \mathbb{R}$ be as in Example 5. Then $\left(\mathscr{A}, \eta^{*}\right)$ is a complete dualistic partial metric space. Define the mapping $T: \mathscr{A} \rightarrow \mathscr{A}$ by

$$
T(\sigma)=\left\{\begin{aligned}
0 & \text { if } \sigma \in\{0,-1,-0.3,-0.1\} \\
-0.3 & \text { if } \sigma=-2 \\
-0.1 & \text { if } \sigma=-3
\end{aligned}\right.
$$

The mapping $T$ satisfies (CCP) for any convergent sequence in $\mathscr{A}$. Indeed, for a convergent sequence $\left\{\sigma_{n}\right\}$ in $\mathscr{A}$ such that $\sigma_{n} \rightarrow \sigma, \sigma \in \mathscr{A}$ due to completeness of $\left(\mathscr{A}, \eta^{*}\right)$. Thus for every such $\sigma$ we have $\eta^{*}(\sigma, \sigma) \leq \eta^{*}(T(\sigma), T(\sigma))$. Observe that for each case (as shown in Table 1) there exist some $a, b, c \geq 0$ with $a+b+c<1$ (however, assuming different values in each case) for which the contractive condition (41) is satisfied.

Example 8. Let $\mathscr{A}=\{0,-1,-2,-3,-0.3,-0.1\} \quad$ and $\eta^{*}: \mathscr{A} \times \mathscr{A} \rightarrow \mathbb{R}$ be as in Example 6. Then $\left(\mathscr{A}, \eta^{*}\right)$ is a complete dualistic partial metric space. Define the mapping
$T: \mathscr{A} \rightarrow \mathscr{A}$ as in Example 7. It is obvious that (3.36) holds for all $\sigma, \varsigma \in \mathscr{A}$, while, the classical Reich fixed point theorem is not applicable. Indeed, for $\sigma=\varsigma=-1$, we have

$$
\begin{aligned}
\eta^{*}(T(\sigma), T(\varsigma)) & =0>-a-b-c \\
& =a \eta^{*}(\sigma, \varsigma)+b \eta^{*}(\sigma, T(\sigma))+c \eta_{\vee}^{*}(\varsigma, T(\varsigma))
\end{aligned}
$$

for all $a, b, c \in(0,1)$.

Corollary 3. Let $(\mathscr{A}, \eta)$ be a complete partial metric space and let $T: \mathscr{A} \rightarrow \mathscr{A}$ be a mapping satisfying

$$
\eta(T(\sigma), T(\varsigma)) \leq a \eta(\sigma, \varsigma)+b \eta(\sigma, T(\sigma))+c \eta(\varsigma, T(\varsigma))
$$

for all $\sigma, \varsigma \in \mathscr{A}$ and $a, b, c \geq 0$ such that $a+b+c<1$. Then, $T$ has a fixed point.

Proof. Since the restriction of a dualistic partial metric $\eta^{*}$ to $[0, \infty),\left.\eta^{*}\right|_{[0, \infty)}=\eta$, is a partial metric, so arguments follow the same lines as in the proof of Theorem 2.

Corollary 4 ([12]). Let $(\mathscr{A}, d)$ be a complete metric space and let $T: \mathscr{A} \rightarrow \mathscr{A}$ be a mapping satisfying

$$
d(T(\sigma), T(\varsigma)) \leq a d(\sigma, \varsigma)+b d(\sigma, T(\sigma))+c d(\varsigma, T(\varsigma))
$$

for all $\sigma, \varsigma \in \mathscr{A}$ and $a, b, c \geq 0$ such that $a+b+c<1$. Then, $T$ has a fixed point.

Proof. Set $\eta(\sigma, \sigma)=0$ for all $\sigma \in \mathscr{A}$, in Corollary 3 .

The following theorem implies the uniqueness of the fixed point of a new Rational type dualistic contraction. 
Theorem 3. Let $\left(\mathscr{A}, \eta^{*}\right)$ be a complete dualistic partial metric space and let $T: \mathscr{A} \rightarrow \mathscr{A}$ be a mapping satisfying (CCP). If there exist $a, b, c \geq 0$ with $a+b+c<1$ such that

$$
\begin{aligned}
\left|\eta^{*}(T(\sigma), T(\varsigma))\right| & \leq a \frac{\left|\eta^{*}(\sigma, T(\sigma)) \cdot \eta^{*}(\varsigma, T(\varsigma))\right|}{\left|\eta^{*}(\sigma, y)\right|} \\
& +b\left|\eta^{*}(\varsigma, T(\varsigma))\right|+c\left|\eta^{*}(\sigma, y)\right|,
\end{aligned}
$$

for all $\sigma, \varsigma \in \mathscr{A}$. Then, $T$ has a fixed point.

Proof. Let $\left\{\sigma_{n}\right\}_{n \in \mathbb{N}}$ be a sequence with an initial point $\sigma_{0} \in \mathscr{A}$ such that $\sigma_{n}=T\left(\sigma_{n-1}\right)$ for all $n \in \mathbb{N}$. If there exists $n_{0} \in \mathbb{N}$ such that $\sigma_{n_{0}}=\sigma_{n_{0}+1}=T\left(\sigma_{n_{0}}\right)$, then $\sigma_{n_{0}}$ is a fixed point of $T$. On the other hand, if $\sigma_{n} \neq \sigma_{n+1}$ for all $n \in \mathbb{N}$, then from contractive condition (64), we have

$$
\begin{aligned}
\left|\eta^{*}\left(\sigma_{n}, \sigma_{n+1}\right)\right|= & \left|\eta^{*}\left(T\left(\sigma_{n-1}\right), T\left(\sigma_{n}\right)\right)\right| \\
\leq & a\left|\frac{\eta^{*}\left(\sigma_{n-1}, T\left(\sigma_{n-1}\right)\right) \cdot \eta^{*}\left(\sigma_{n}, T\left(\sigma_{n}\right)\right)}{\eta^{*}\left(\sigma_{n-1}, \sigma_{n}\right)}\right| \\
& +b\left|\eta^{*}\left(\sigma_{n}, T\left(\sigma_{n}\right)\right)\right|+c\left|\eta^{*}\left(\sigma_{n-1}, \sigma_{n}\right)\right| \\
= & a\left|\frac{\eta^{*}\left(\sigma_{n-1}, \sigma_{n}\right) \cdot \eta^{*}\left(\sigma_{n}, \sigma_{n+1}\right)}{\eta^{*}\left(\sigma_{n-1}, \sigma_{n}\right)}\right| \\
& +b\left|\eta^{*}\left(\sigma_{n}, \sigma_{n+1}\right)\right|+c\left|\eta^{*}\left(\sigma_{n-1}, \sigma_{n}\right)\right| \\
= & (a+b)\left|\eta^{*}\left(\sigma_{n}, \sigma_{n+1}\right)\right|+c\left|\eta^{*}\left(\sigma_{n-1}, \sigma_{n}\right)\right| \\
\left|\eta^{*}\left(\sigma_{n}, \sigma_{n+1}\right)\right| \leq & \frac{c}{1-a-b}\left|\eta^{*}\left(\sigma_{n-1}, \sigma_{n}\right)\right| .
\end{aligned}
$$

Let $\lambda=c /(1-a-b)<1$, so

$\left|\eta^{*}\left(\sigma_{n}, \sigma_{n+1}\right)\right| \leq \lambda\left|\eta^{*}\left(\sigma_{n-1}, \sigma_{n}\right)\right| \leq \lambda^{2}\left|\eta^{*}\left(\sigma_{n-2}, \sigma_{n-1}\right)\right| \cdots \lambda^{n}\left|\eta^{*}\left(\sigma_{0}, \sigma_{1}\right)\right|$.

Now,

$$
\begin{aligned}
\left|\eta^{*}\left(\sigma_{n}, \sigma_{n}\right)\right|= & \left|\eta^{*}\left(T\left(\sigma_{n-1}\right), T\left(\sigma_{n-1}\right)\right)\right| \\
\leq & a\left|\frac{\eta^{*}\left(\sigma_{n-1}, T\left(\sigma_{n-1}\right)\right) \cdot \eta^{*}\left(\sigma_{n-1}, T\left(\sigma_{n-1}\right)\right)}{\eta^{*}\left(\sigma_{n-1}, \sigma_{n-1}\right)}\right| \\
& +b\left|\eta^{*}\left(\sigma_{n-1}, T\left(\sigma_{n-1}\right)\right)\right|+c\left|\eta^{*}\left(\sigma_{n-1}, \sigma_{n-1}\right)\right| \\
= & a\left|\frac{\eta^{*}\left(\sigma_{n-1}, \sigma_{n}\right) \cdot \eta^{*}\left(\sigma_{n-1}, \sigma_{n}\right)}{\eta^{*}\left(\sigma_{n-1}, \sigma_{n-1}\right)}\right| \\
& +b\left|\eta^{*}\left(\sigma_{n-1}, \sigma_{n}\right)\right|+c\left|\eta^{*}\left(\sigma_{n-1}, \sigma_{n-1}\right)\right| .
\end{aligned}
$$

$\operatorname{As} \eta^{*}\left(\sigma_{n-1}, \sigma_{n}\right) / \eta^{*}\left(\sigma_{n-1}, \sigma_{n-1}\right) \geq 1$, we get

$$
\begin{aligned}
\left|\eta^{*}\left(\sigma_{n}, \sigma_{n}\right)\right| & \leq a\left|\eta^{*}\left(\sigma_{n-1}, \sigma_{n}\right)\right|+b\left|\eta^{*}\left(\sigma_{n-1}, \sigma_{n}\right)\right|+c\left|\eta^{*}\left(\sigma_{n-1}, \sigma_{n-1}\right)\right| \\
& =(a+b)\left|\eta^{*}\left(\sigma_{n-1}, \sigma_{n}\right)\right|+c\left|\eta^{*}\left(\sigma_{n-1}, \sigma_{n-1}\right)\right| \\
& =(a+b) \lambda^{n-1}\left|\eta^{*}\left(\sigma_{0}, \sigma_{1}\right)\right|+c\left|\eta^{*}\left(\sigma_{n-1}, \sigma_{n-1}\right)\right| .
\end{aligned}
$$

Arguing like above, we have

$$
\left|\eta^{*}\left(\sigma_{n-1}, \sigma_{n-1}\right)\right| \leq(a+b) \lambda^{n-2}\left|\eta^{*}\left(\sigma_{0}, \sigma_{1}\right)\right|+c\left|\eta^{*}\left(\sigma_{n-2}, \sigma_{n-2}\right)\right| .
$$

The inequality (68) leads to $\left|\eta^{*}\left(\sigma_{n}, \sigma_{n}\right)\right| \leq(a+b)\left(\lambda^{n-1}+c \lambda^{n-2}\right)\left|\eta^{*}\left(\sigma_{0}, \sigma_{1}\right)\right|+c^{2}\left|\eta^{*}\left(\sigma_{n-2}, \sigma_{n-2}\right)\right|$

Continuing in the same way, we get

$$
\begin{aligned}
\left|\eta^{*}\left(\sigma_{n}, \sigma_{n}\right)\right| \leq & (a+b)\left(\lambda^{n-1}+c \lambda^{n-2}+c^{2} \lambda^{n-3}+\cdots+c^{n-1}\right) \\
& \cdot\left|\eta^{*}\left(\sigma_{0}, \sigma_{1}\right)\right|+c^{n}\left|\eta^{*}\left(\sigma_{0}, \sigma_{0}\right)\right|, \\
\left|\eta^{*}\left(\sigma_{n}, \sigma_{n}\right)\right| \leq & (a+b)\left(\frac{c^{n-1}-\lambda^{n-1}}{c-\lambda}\right)\left|\eta^{*}\left(\sigma_{0}, \sigma_{1}\right)\right|+c^{n}\left|\eta^{*}\left(\sigma_{0}, \sigma_{0}\right)\right| .
\end{aligned}
$$

The equation (1) implies

$$
\begin{aligned}
d_{\eta^{*}}\left(\sigma_{n}, \sigma_{n+1}\right) \leq & \left|\eta^{*}\left(\sigma_{n}, \sigma_{n+1}\right)\right|+\left|\eta^{*}\left(\sigma_{n}, \sigma_{n}\right)\right| \\
\leq & \lambda^{n}\left|\eta^{*}\left(\sigma_{0}, \sigma_{1}\right)\right|+(a+b)\left(\frac{\lambda^{n-1}-c^{n-1}}{\lambda-c}\right)\left|\eta^{*}\left(\sigma_{0}, \sigma_{1}\right)\right| \\
& +c^{n}\left|\eta^{*}\left(\sigma_{0}, \sigma_{0}\right)\right| \\
\leq & \left(\lambda^{n}+\lambda^{n-2}+c \lambda^{n-3}+\cdots+c^{n-2}\right)\left|\eta^{*}\left(\sigma_{0}, \sigma_{1}\right)\right| \\
& +c^{n}\left|\eta^{*}\left(\sigma_{0}, \sigma_{0}\right)\right| .
\end{aligned}
$$

Set $\lambda^{n}+\lambda^{n-2}+c \lambda^{n-3}+\cdots+c^{n-2}=\gamma^{n}$. Then,

$$
d_{\eta^{*}}\left(\sigma_{n}, \sigma_{n+1}\right) \leq \gamma^{n}\left|\eta^{*}\left(\sigma_{0}, \sigma_{1}\right)\right|+c^{n}\left|\eta^{*}\left(\sigma_{0}, \sigma_{0}\right)\right| .
$$

Now, for $m>n$, we have

$$
\begin{aligned}
d_{\eta^{*}}\left(\sigma_{n}, \sigma_{m}\right) \leq & d_{\eta^{*}}\left(\sigma_{n}, \sigma_{n+1}\right)+d_{\eta^{*}}\left(\sigma_{n+1}, \sigma_{n+2}\right)+\cdots+d_{\eta^{*}}\left(\sigma_{m-1}, \sigma_{m}\right) \\
\leq & \gamma^{n}\left|\eta^{*}\left(\sigma_{0}, \sigma_{1}\right)\right|+c^{n}\left|\eta^{*}\left(\sigma_{0}, \sigma_{0}\right)\right|+\gamma^{n+1}\left|\eta^{*}\left(\sigma_{0}, \sigma_{1}\right)\right| \\
& +c^{n+1}\left|\eta^{*}\left(\sigma_{0}, \sigma_{0}\right)\right|+\cdots+\gamma^{m-1}\left|\eta^{*}\left(\sigma_{0}, \sigma_{1}\right)\right|+c^{m-1}\left|\eta^{*}\left(\sigma_{0}, \sigma_{0}\right)\right| \\
\leq & \left(\gamma^{n}+\gamma^{n+1}+\cdots+\gamma^{n+1}\right)\left|\eta^{*}\left(\sigma_{0}, \sigma_{1}\right)\right| \\
& +\left(c^{n}+c^{n+1}+\cdots+c^{m-1}\right)\left|\eta^{*}\left(\sigma_{0}, \sigma_{1}\right)\right| .
\end{aligned}
$$

We deduce that $\lim _{n, m \rightarrow \infty} d_{\eta^{*}}\left(\sigma_{n}, \sigma_{m}\right)=0$ and similarly, $\lim _{n, m \rightarrow \infty} d_{\eta^{*}}\left(\sigma_{m}, \sigma_{n}\right)=0 \quad$ which implies that $\lim _{n, m \rightarrow \infty} d_{\eta^{*}}^{s}\left(\sigma_{n}, \sigma_{m}\right)=0$. The remaining part of this proof is similar to the proof of Theorem 2 .

Corollary 5. Let $(\mathscr{A}, \eta)$ be a complete partial metric space and let $T: \mathscr{A} \rightarrow \mathscr{A}$ be a mapping satisfying

$\eta(T(\sigma), T(\varsigma)) \leq a \frac{\eta(\sigma, T(\sigma)) \cdot \eta(\varsigma, T(\varsigma))}{\eta(\sigma, y)}+b \eta(\varsigma, T(\varsigma))+c \eta(\sigma, y)$,

for all $\sigma, \varsigma \in \mathscr{A}$ and $a, b, c$ are non negative numbers such that $a+b+c<1$. Then, $T$ has a fixed point.

Proof. Since the restriction of a dualistic partial metric $\eta^{*}$ to $[0, \infty),\left.\eta^{*}\right|_{[0, \infty)}=\eta$ is a partial metric, the result is obvious. $\square$

Corollary 6. Let $(\mathscr{A}, d)$ be a complete metric space and let $T: \mathscr{A} \rightarrow \mathscr{A}$ be a mapping satisfying

$d(T(\sigma), T(\varsigma)) \leq a \frac{d(\sigma, T(\sigma)) \cdot d(\varsigma, T(\varsigma))}{d(\sigma, \varsigma)}+b d(\varsigma, T(\varsigma))+c d(\sigma, \varsigma)$ 
for all $\sigma, \varsigma \in \mathscr{A}$ and $a, b, c$ are non negative numbers such that $a+b+c<1$. Then, $T$ has a fixed point.

Proof. Take in Corollary $5, \eta(\sigma, \sigma)=0$ for all $\sigma \in \mathscr{A}$.

\section{Application}

In this section, we present an application of Theorem 1 to ensure the existence of the solution of the boundary value problem given by

$$
\begin{aligned}
-\frac{d^{2} \sigma}{d t^{2}} & =f(t, \sigma(t)), \quad t \in[0,1], \\
\sigma(0) & =\sigma(1)=0,
\end{aligned}
$$

where $f:[0,1] \times \mathbb{R} \rightarrow \mathbb{R}$ is a continuous mapping. The Green function associated to the boundary value problem (78) is defined by

$$
V(t, \tau)= \begin{cases}t(1-\tau), & 0 \leq t \leq \tau \leq 1, \\ \tau(1-t), & 0 \leq \tau \leq t \leq 1 .\end{cases}
$$

Let $C[0,1]$ be the space of all continuous mappings defined on $[0,1]$. Let $\mathscr{A}=(C[0,1], \mathbb{R})$. Define the mapping $\eta^{*}: \mathscr{A} \times \mathscr{A} \rightarrow \mathbb{R}$ by

$$
\eta^{*}(\sigma, \varsigma)=\|(\sigma-\varsigma)\|_{\infty}+L=\sup _{t \in[0,1]}|\sigma(t)-\varsigma(t)|+L, L \in \mathbb{R} .
$$

It is known that $\left(\mathscr{A}, \eta^{*}\right)$ is a complete dualistic partial metric space. Define $T: \mathscr{A} \rightarrow \mathscr{A}$ by

$$
T \sigma(t)=\int_{0}^{1} V(t, \tau) f(\tau, \sigma(\tau)) d \tau
$$

for all $t \in[0,1]$. Note that the problem (78) has a solution iff the operator $T$ has a fixed point.

Theorem 4. Let $\mathscr{A}=C([0,1], \mathbb{R})$. Define the mapping $T: \mathscr{A} \rightarrow \mathscr{A}$ by

$$
T \sigma(t)=\int_{0}^{1} V(t, \tau) f(\tau, \sigma(\tau)) d \tau,
$$

where $f:[0,1] \times \mathscr{A} \rightarrow \mathbb{R}$ is a continuous mapping and $\sigma(t)$ is such that $\eta^{*}(\sigma(t), \sigma(t)) \leq \eta^{*}(T(\sigma(t))), T(\sigma(t))$. Assume that

(i) The mapping $f:[0,1] \times \mathscr{A} \rightarrow \mathbb{R}$ satisfies

$$
|f(t, \sigma)-f(t, \varsigma)| \leq 8 \ln \left(\frac{e^{k \omega}}{\rho}\right)
$$

for all $t \in[0,1], \sigma, \varsigma \in \mathscr{A}, \omega=\max \left\{\left|\eta^{*}(\sigma, T(\sigma))\right|,\left|\eta^{*}(\varsigma, T(\varsigma))\right|\right\}$ and $\rho>0$;

Then, boundary value problem (78) has a solution.

Proof. We note that $q(t) \in\left(C^{2}[0,1], \mathbb{R}\right)$ (say) is a solution of (78) if and only if $q(t) \in \mathscr{A}$ is a solution of the integral equation (82). The solution of (82) is given by the fixed point of $T$, i.e., $q(t)=T(q(t))$.
Let $\sigma, \varsigma \in \mathscr{A}$ and $t \in[0,1]$, by assumption (i), we get

$$
\begin{aligned}
|T \sigma(t)-T \varsigma(t)| & =\left[\left|\int_{0}^{1} V(t, \tau)[f(\tau, \sigma(\tau))-f(\tau, \varsigma(\tau))] d \tau\right|\right] \\
& \leq\left[\int_{0}^{1} V(t, \tau)|f(\tau, \sigma(\tau))-f(\tau, \varsigma(\tau))| d \tau\right] \\
& \leq\left[8 \int_{0}^{1} V(t, \tau) \ln \left(\frac{e^{k \omega}}{\rho}\right) d \tau\right] \\
& \leq\left[8 \int_{0}^{1} V(t, \tau) \ln \left(\frac{e^{k \omega}}{\rho}\right) d \tau\right] \\
& =8 \ln \left(\frac{e^{k \omega}}{\rho}\right)\left(\sup _{t \in[0,1]}\left[\int_{0}^{1} V(t, \tau) d \tau\right]\right) .
\end{aligned}
$$

Since $\int_{0}^{1} V(t, \tau) d \tau=-\left(t^{2} / 2\right)+(t / 2)$ for all $t \in[0,1]$, we have $\sup _{t \in[0,1]}\left[\int_{0}^{1} V(t, \tau) d \tau\right]=1 / 8$, which implies that

$$
\begin{aligned}
\left|\eta^{*}(T(\sigma), T(\varsigma))\right| & =\sup _{t \in[0,1]}|T \sigma(t)-T \varsigma(t)|+L \leq k \omega, \text { where } L=\ln (\rho) \\
& =k \max \left\{\left|\eta^{*}(\sigma, T(\sigma))\right|,\left|\eta^{*}(\varsigma, T(\varsigma))\right|\right\} .
\end{aligned}
$$

Hence, application of Theorem 1 ensures that $T$ has at least one fixed point $q(t) \in \mathscr{A}$, that is, $T(q(t))=q(t)$ which is a solution of (82).

\section{Data Availability}

The data used to support the findings of this study are available from the corresponding author upon request.

\section{Conflicts of Interest}

The authors declare that they have no conflicts of interest.

\section{Authors' Contributions}

All authors contributed equally and significantly in writing this article. All authors read and approved the final manuscript.

\section{Acknowledgments}

The third author would like to thank Prince Sultan University for funding this work through research group Nonlinear Analysis Methods in Applied Mathematics (NAMAM) group number RG-DES-2017-01-17.

\section{References}

[1] H. Aydi, E. Karapinar, and A. F. Rold án López de Hierro, " $\omega$-Interpolative Ciric-Reich-Rus type contractions," Mathematics, vol. 7, no. 1, p. 57, 2019.

[2] E. Karapinar, R. P. Agarwal, and H. Aydi, "Interpolative Reich-Rus-Ciric type contractions on partial metric spaces," Mathematics, vol. 6, p. 256, 2018. 
[3] D. W. Boyd and J. S. W. Wong, "On nonlinear contractions," Proceedings of the American Mathematical Society, vol. 20, pp. 458-464, 1969.

[4] J. Caristi, "Fixed point theorem for mapping satisfying inwardness conditions," Transactions of the American Mathematiacl Society, vol. 215, pp. 241-251, 1976.

[5] L. B. Círíc, "A generalization of Banach's contraction principle," Proceedings of the American Mathematical Society, vol. 45, no. 2, pp. 267-273, 1974.

[6] M. Geraghty, "On contraction mappings," Proceedings of the American Mathematical Society, vol. 40, pp. 604-608, 1973.

[7] J. R. Jachymski, "Equivalence of some contractivity properties over metrical structure," Proceedings of the American Mathematical Society, vol. 125, pp. 2327-2335, 1997.

[8] W. A. Kirk, "Fixed points of asymptotic contractions," Journal of Mathematical Analysis and Applications, vol. 277, no. 2, pp. 645-650, 2003.

[9] A. Meir and E. Keeler, "A theorem on contraction mappings," Journal of Mathematical Analysis and Applications, vol. 28, no. 2, pp. 326-329, 1969.

[10] S. B. Nadler, "Multivalued contraction mappings," Pacific Journal of Mathematics, vol. 30, no. 2, pp. 475-488, 1969.

[11] E. Rakotch, "A note on contractive mappings," Proceedings of the American Mathematical Society, vol. 13, no. 3, pp. 459-459, 1962.

[12] S. Reich, "Some remarks concerning contraction mappings," Canadian Mathematical Bulletin, vol. 14, no. 1, pp. 121-124, 1971.

[13] B. E. Rhoades, "Some theorems on weakly contractive maps," Nonlinear Analysis: Theory, Methods \& Applications, vol. 47, no. 4, pp. 2683-2693, 2001.

[14] S. Romaguera, "Fixed point theorems for generalized contractions on partial metric spaces," Topology and its Applications, vol. 218, pp. 2398-2406, 2011.

[15] M. C. B. Reurings and A. C. M. Ran, "A fixed point theorem in partially ordered sets and some applications to matrix equations," Proceedings of the American Mathematical Society, vol. 132, pp. 1435-1443, 2004.

[16] W. Shatanawi, A. Al-Rawashdeh, H. Aydi, and H. K. Nashine, "On a fixed point for generalized contractions in generalized metric spaces," Abstract and Applied Analysis, vol. 2012, Article ID 246085, 13 pages, 2012.

[17] T. Suzuki, "A generalized Banach contraction principle that characterizes metric completeness," Proceedings of the American Mathematical Society, vol. 136, no. 5, pp. 1861-1870, 2008.

[18] S. G. Matthews, "Partial metric topology," Annals of the New York Academy of Sciences, vol. 728, pp. 183-197, 1994.

[19] M. A. Alghamdi, N. Shahzad, and O. Valero, "On fixed point theory in partial metric spaces," Fixed Point Theory and Applications, vol. 2012, no. 1, p. 175, 2012.

[20] H. Aydi, S. H Amor, and E. Karapinar, "Berinde-type generalized contractions on partial metric spaces," Abstract and Applied Analysis, vol. 2012, Article ID 312479, p. 10, 2013.

[21] M. Nazam, M. Arshad, and C. Park, "Fixed point theorems for improved $\alpha$-geraghty contractions in partial metric spaces," Journal of Nonlinear Sciences and Applications, vol. 09, no. 06, pp. 4436-4449, 2016.

[22] S. J. O'Neill, "Partial metric, valuations and domain theory," Annals of the New York Academy of Sciences, vol. 806, pp. 304-315, 1996.
[23] S. Oltra and O. Valero, "Banach's fixed point theorem for partial metric spaces," Rendiconti dell'Istituto di Matematica dell'Università di Trieste, vol. 36, pp. 17-26, 2004.

[24] M. Nazam, M. Arshad, and M. Abbas, "Some fixed point results for dualistic rational contractions," Applied General Topology, vol. 17, no. 2, pp. 199-209, 2016.

[25] M. Nazam and M. Arshad, "Some fixed point results in ordered dualistic partial metric spaces," Transactions of A. Razmadze Mathematical Institute, vol. 172, no. 3, pp. 498-509, 2018.

[26] R. M. T. Bianchini, "Su un problema di S. Reich riguardante la teoria dei punti fissi," Bollettino dellUnione Matematica Italiana, vol. 5, pp. 103-108, 1972.

[27] B. K. Dass and S. Gupta, "An extension of Banach contraction principle through rational expressions," Indian Journal of Pure and Applied Mathematics, vol. 6, pp. 1455-1458, 1975. 


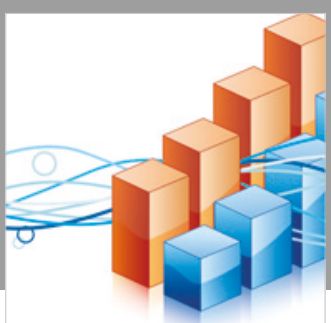

Advances in

Operations Research

\section{-n-m}
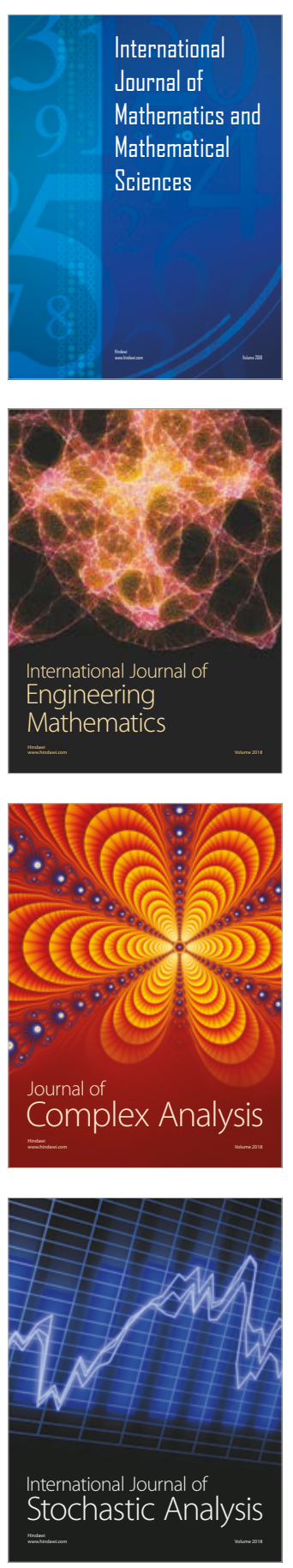
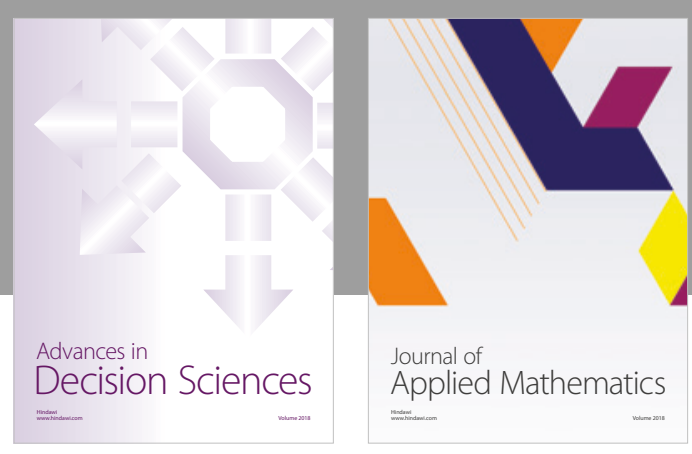

Journal of

Applied Mathematics
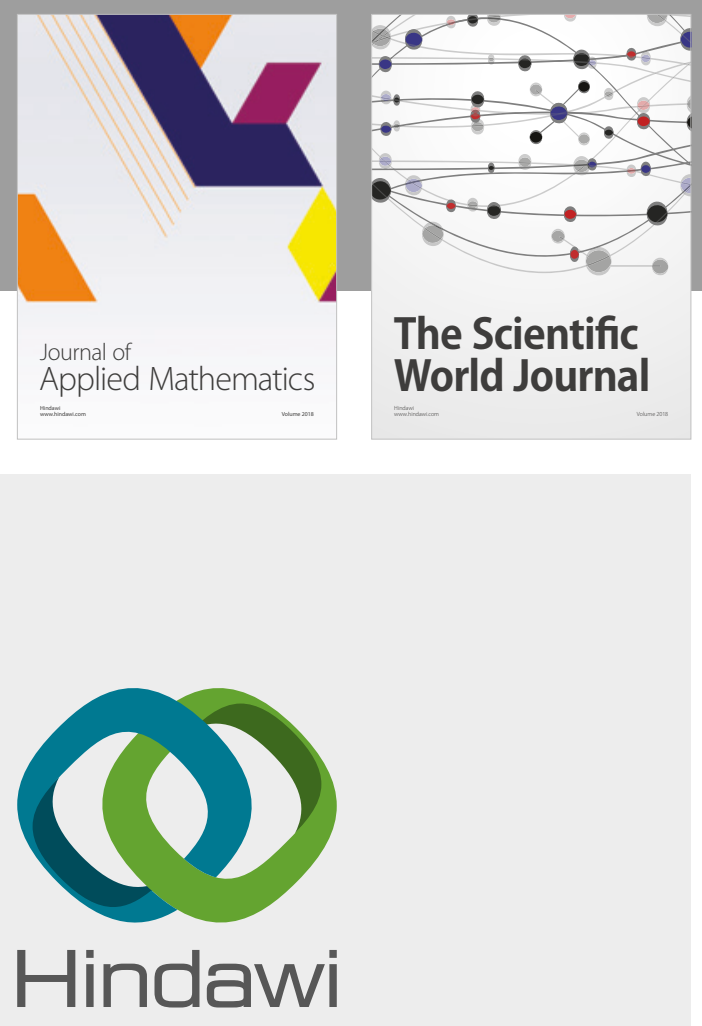

Submit your manuscripts at

www.hindawi.com

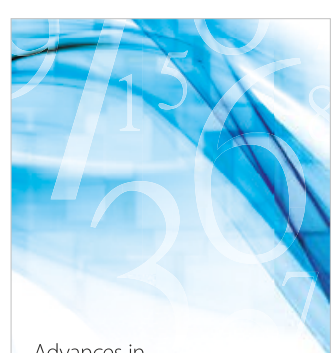

Advances in
Numerical Analysis
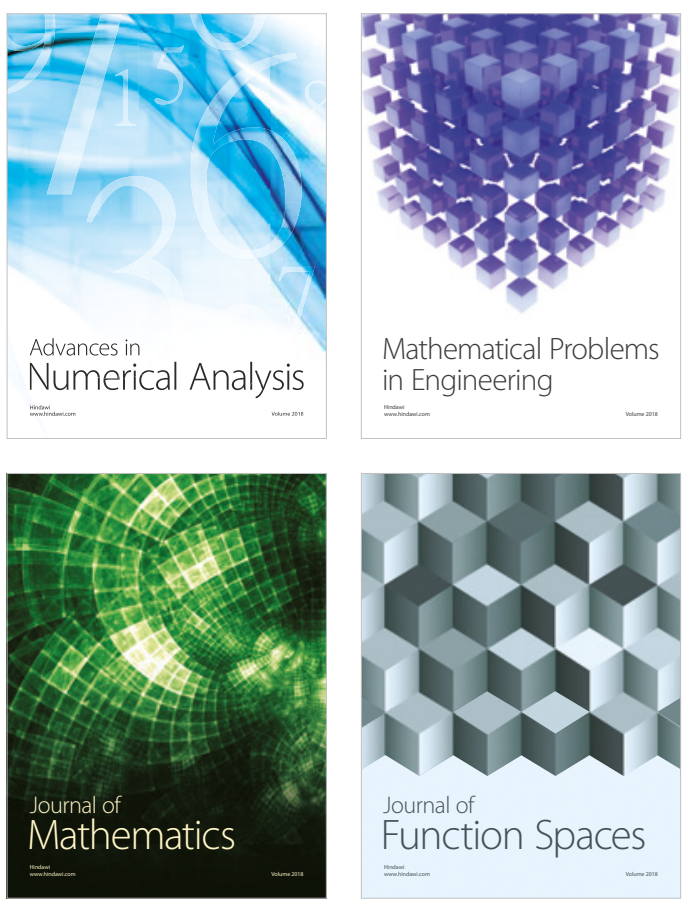

Mathematical Problems in Engineering

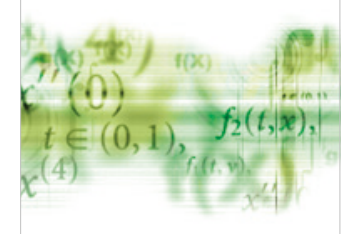

International Journal of

Differential Equations

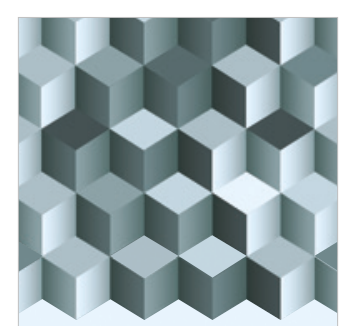

Journal of

Function Spaces
The Scientific

World Journal

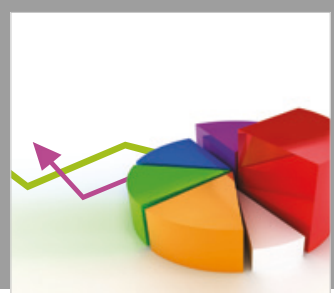

Journal of

Probability and Statistics
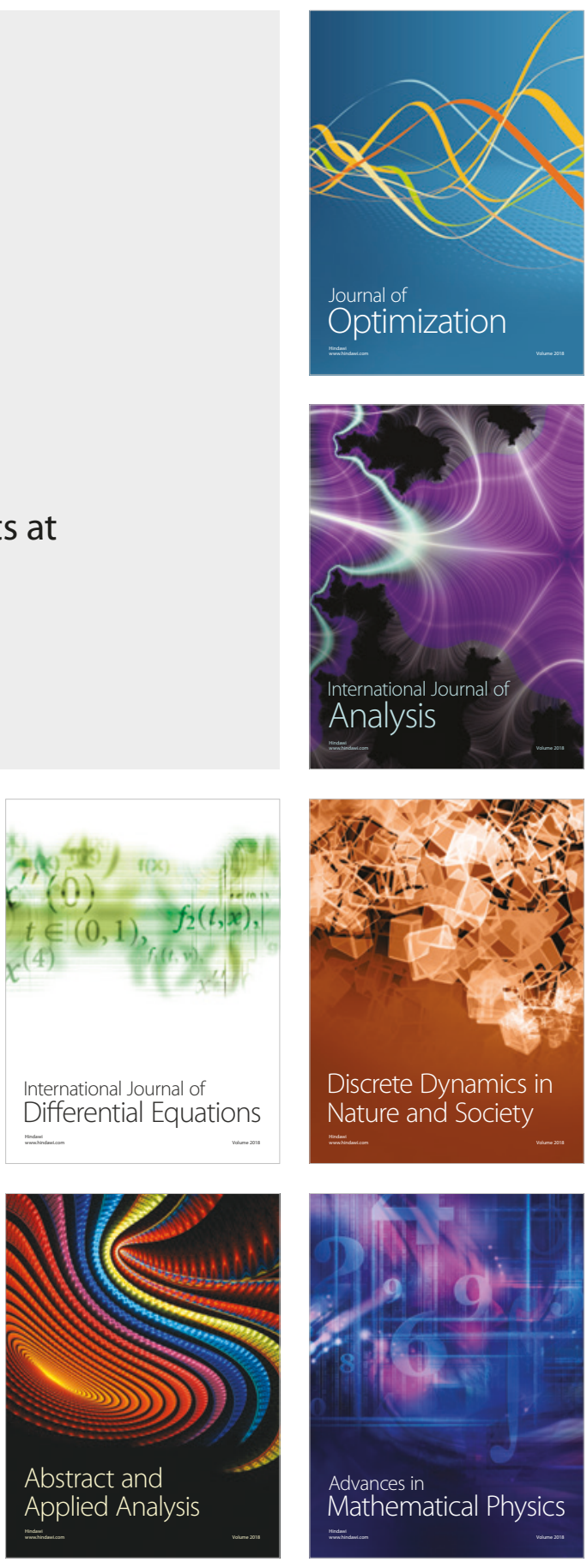